\title{
Design of short low speed Göttingen type wind tunnel: CFD simulation
}

\author{
Hardy Weisweiler $^{1}$, Jasmina Kojouharova ${ }^{1,{ }^{*}}$, and Roland Dückershoff ${ }^{1}$ \\ ${ }^{1}$ Department of Mechanical Engineering, Mechatronics and Materials Technology, \\ THM University of Applied Sciences, 61169 Friedberg, Germany
}

\begin{abstract}
The paper is focused on design simulations by the means of commercial software for a low speed circuit wind tunnel with an open test section, recently built at the Department of Mechanical Engineering, Mechatronics and Materials Technology at THM University of Applied Sciences in Friedberg. The proposed wind tunnel has a test section with cross sectional area of $1 \mathrm{X} 1 \mathrm{~m}^{2}$ and a length of $1.6 \mathrm{~m}$. The maximum achievable speed is about $50 \mathrm{~m} / \mathrm{s}$ with empty test section. The simulation had the target to propose and to verify various geometries - test chamber, tunnel contraction, diffuser etc. as well the tunnel corner including the vanes. In addition, the influence of the vanes number and their shape on the ventilator power needed and the flow velocity uniformity as well, have been subjects of the investigation. Also the ventilator type (rotational segment) impact on the flow pattern within the operational area has been scrutinized.
\end{abstract}

\section{Introduction}

Wind tunnels generate nearly uniform air flows, with low turbulence intensity and have the advantage to test the flow properties under well controlled conditions compared to experiments in the open environment. These devices have been in operation since the end of the 19th century and could be built by two different manners: closed loop Gottingen type respectively open Eiffel type wind tunnel (s. Fig. 1). They can be further divided into open and close test section types.

The wind tunnel can be classified also by flow speed into four groups: (i) subsonic or low-speed; (ii) transonic; (iii) supersonic; (iv) hypersonic.

At low flow speed, due to the dynamic similarity, the same Reynolds numbers as for the real application are required in order to enhance the reliability of the test data. The scale of the test models is predefined by the test chamber size and since the almost all wind tunnels operate at the air atmospheric pressure, the only option left is to increase the flow velocity. Therefore, achieving of high flow velocity has been sought.

When talking about computational engineering, it is meant that we attempt to "mimic" the nature, to describe the real processes by means of conservation equations or in other words to fix the mathematical model and then playing with the parameters to learn more about the processes there.

\footnotetext{
*Corresponding author: jasmina.kojouharova@m.thm.de
} 
Here the questions are: first - how realistic we describe the physical processes and second - how reliable the computations are. Certainly, the experimental studies should run, especially at high Reynolds number, for full identification of the flow characteristics and thus to validate the numerical simulations. In this aspect even if the velocity of interest is out of the windtunnel operation range, the fluid flow simulations still can be validated if no other physical effects are met. Therefore, the wind-tunnel experiments and CFD simulations might be considered as complementary ones.
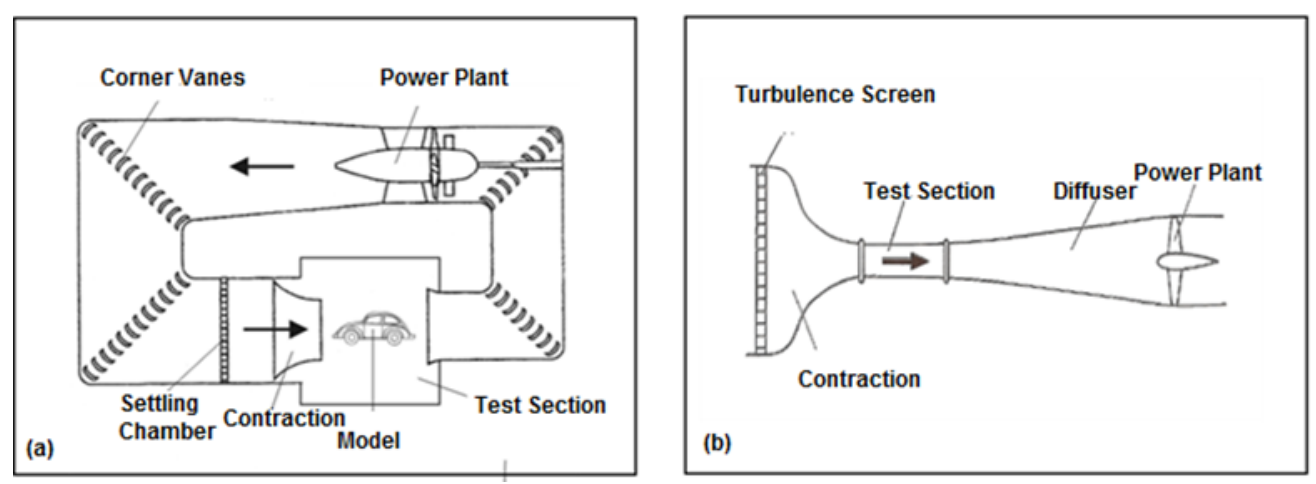

Fig. 1. Schematic of (a) closed loop Göttingen type wind tunnel (b) open Eiffel type wind tunnel

A wind tunnel is characterised by the flow quality inside the test chamber. The drawback of that devices is that the flow field could not be well developed alongside the tunnel, especially in the case of short one. Moreover, the side's effects could not be completely excluded and thus also they affect the flow characteristics.

A compromise has to be made and a wind tunnel with a flow uniformity within the range $0.5-2 \%$, the turbulence intensity of $0.5-2 \%$ is considered as a good one. It should provide temperature uniformity within $0.1-0.5 \%$ at the inlet of the test section.

The design of the wind tunnels is a complex task involving fluid mechanics issues and typical engineering aspects. Nevertheless, the topic is well discussed in the literature (for instance, the already classical publication [1-2] or the recent reports [3-4]). However, some concerns still remain to be clarified due to the fact that the design depends mainly on the wind tunnel final purpose. In addition some restrictions, like for instance the limited space available for the wind tunnel, makes difficulties to achieve the flow quality desired. It requires an innovative design to be studied in order to keep the test section within the specification: a cross sectional area of $1 \times 1 \mathrm{~m}^{2}$ and a length of $1.6 \mathrm{~m}$.

Due to specific infrastructure reason we have faced also severe electric power restriction. So that the accurate assessment of the losses along the tunnel is compulsory.

\section{Wind tunnel design considerations}

At first, the decision has been taken in favour of a closed circuit wind tunnel. Besides of the infrastructural circumstances in this case, due to kinetic energy conservation of the airflow, typically less power is needed for reaching the velocity required, although the general design complexity evokes more difficulties.

The overall layout of the proposed wind tunnel is shown in Fig. 2. The airflow circulates counter clockwise (s. Fig. 2) from the suction zone at the end of the test camera through diffuser, settling chambers and contraction being driven through the power plant. 
Based on long lasting experience, the E.T.S.I. group at the Madrid Technical University [4] elaborates a methodology focused on the reduction of construction and operation cost of low speed wind tunnel (LSWT) for a given performance and quality requirement. Thereafter they implemented it in an Excel spreadsheet (web page htts://www.aero.upm.es/LSLCWT) as an assistance of new wind tunnel designers.
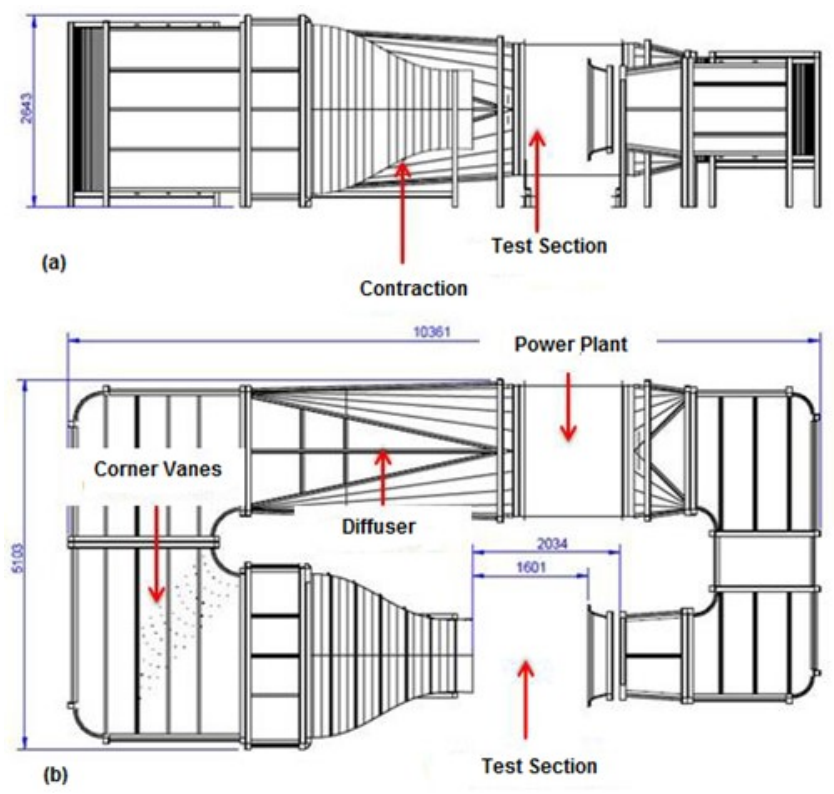

Fig. 2. Layout of the THM wind tunnel: (a) vertical intersection (b) longitudinal intersection.

Following the recommendations with the specification given: maximum achievable velocity of $50 \mathrm{~m} / \mathrm{s}$ and the prescribed test section dimensions, we got the LSWT length of $13.1 \mathrm{~m}$ and width of $5.4 \mathrm{~m}$ far above the specification of $10.5 \mathrm{~m}$ length and $4.5 \mathrm{~m}$ width.

The possible way to reduce the overall size is to decrease the contraction ratio, i.e. the ratio between the largest cross section area, found in the stagnation chamber and the smallest cross section area found in the test section. However, a high contraction ratio is a key factor in achieving a good quality flow. Another way is to use the wide angle diffusers which may lead to flow detachment. It is crucial to avoid flow separation close to the wall of the contraction zone. Separations may cause vibrations which are also undesirable.

The corners with the incorporate turning vanes could sufficiently improve the flow quality. The parameters to be considered in the design are the radius, the spacing between vanes and the flow impact angle. The use of stagnation chamber with honeycomb and screen layer leads to decrease the turbulence intensity.

At the stage of design, the most adequate method to verify that design meets those criteria is computational fluid dynamics (CFD).

\section{Physical model}

Numerical simulations have been performed for the 3D turbulent incompressible flow built on a Reynolds Average Navier-Stokes (RANS) steady state calculations by $\kappa-\varepsilon$ turbulence model. The development in the time the flow is not a matter of discussion in this paper. 
Due to the complex geometry of the construction (s. Fig. 2) some simplification have been implemented in order to keep the calculation time at some reasonable limits. In the subdomain, representing the impeller, a set of virtual forces is applied, which replace the modelling of the axial fan blades and produce similar effects in the outer flow space.

3D fan zones, considered as fluid cell zones, simulate the effect of an axial fan by applying a distributed momentum source in a toroid-shape fluid volume. The axial and the swirl momentum sources are expressed as:

$$
\begin{gathered}
S_{a x}\left[\mathrm{~kg} \mathrm{~m}^{-2} \mathrm{~s}^{-2}\right]=\frac{\Delta p}{\Delta x} \\
S_{\text {swirl }}\left[\mathrm{kg} \mathrm{m}^{-2} \mathrm{~s}^{-2}\right]=\frac{\dot{m}}{A_{\text {fan }}} \cdot \frac{1}{\Delta x} \cdot r \cdot \omega
\end{gathered}
$$

The parameters needed are the pressure jump $\Delta p$ in the flow direction and the swirl components, both expressed in relation to the flow rate. In most of the cases the swirl components are not included: the fan characteristics are then solely represented by the pressure jump. This value is typically obtained from experimentally generated fan blades curve. In the formulae above $\Delta x$ stays for the fan thickness, $A_{\text {fan }}$ for the fan cross sectional area, $\dot{m}$ for the mass flow rate, $r$ is the fan radius and $\omega$ the angular velocity.

The settling chamber is located just before the stream entering the nozzle and thus has a major influence on the flow quality in the test section. The THM wind tunnel is equipped with one honeycomb and three screens.

The honeycomb layer has a thickness of $18 \mathrm{~mm}$ and the cylindrical shaped cells are with a diameter of $2.5 \mathrm{~mm}$ and a wall thickness is $0.2 \mathrm{~mm}$. Two of the screens have the thickness of $140 \mu \mathrm{m}$ and one of them is with the thickness of $70 \mu \mathrm{m}$. Their porosity is $68 \%, 49 \%$ and $58 \%$ respectively.

To minimise the computational power needed, the topology of the settling chamber has been neglected and the simulations have been performed under the assumptions that the volume there is empty. While the honeycombs essentially serve very efficiently to reduce the lateral turbulence and the screens attenuate the longitudinal one, the simulated velocity uniformity is to be considered as a low limit. In addition the pressure losses through this chamber has been analytically evaluated.

\section{Results and discussion}

First and foremost, the pressure losses through all the wind tunnel components have been analytical evaluated, however it is beyond the scope of this paper and are not to be discussed now.

To find correlations between the geometry proposed and the pressure losses, only the axial driving forces have been considered initially. This is reasonable because the fan could be equipped with additional grids in order to reduce the swirl momentum forces.

At first, the primary CAD model has been simulated, critically studied and the impact of the particular components has been figured out. Different layouts have been proven to keep the outer dimensions - length, width and high, within the specification given.

Two main simulations targets - to keep the total pressure loss minimal and to look for the best fluid flow performance within the test section, are in some sense contradictory, though the quality criteria are considered as prevailing ones.

The contraction ratio between 4 and 6 is considered as sufficient in the case of wind tunnels for civil or industrial application. In our design the contraction ratio was set to 5.1.

The flow quality is strongly dependent on the geometry of the diffuser just after the power plant. Its length was fixed to $2.9 \mathrm{~m}$ keeping its semi-open angle less than $3.5^{\circ}$. 
The outer dimensions of the corner chamber and the corner radius as well also have been selected. The corner radius is proportional to the chamber entrance so that it was set to be 0.3 $\mathrm{m}$ for the front und $0.4 \mathrm{~m}$ for the back chambers.

The corner with the incorporated turning vanes are accountable for flow structure and for the major part of the total pressure loss, often more than $50 \%$ [4]. Thus, the main simulations issue targets the defining of the inner corner topology.

In order to keep the construction expenses at reasonable level, the vanes have been defined as simple curved plates and a special attention has been draft on the vanes spacing, arc length and their orientation as well.

Two different scenarios have been simulated: distance of $0.2 \mathrm{~m}$ respectively $0.15 \mathrm{~m}$ within the front chambers and $0.25 \mathrm{~m}$ respectively $0.18 \mathrm{~m}$ within the back chambers. It has been proven that the increase of vanes number proposed, results in an increase of the pressure loss their within $10 \%$ which is still tolerable.

Initially, very short vanes, less than $0.1 \mathrm{~m}$, have been considered, which turn out to be ineffective. Then different type of arcs (length and attack angle) have been simulated and the result, relevant to the arc with an angle of $96 \mathrm{o}$, has been found to be the most efficient one. The arc lengths have been fixed to $0.51 \mathrm{~m}$ for front chambers and $0.67 \mathrm{~m}$ for the back chambers.

The variety of design layouts has been examined. Here we are not to follow all of them and further the result only for the design selected, which might be identified in Fig. 4, are presented.

The pressure losses as a function of the volume flow rate have been studied and the results are summarised in Table 1.

Table 1. Pressure losses within the wind tunnel in dependence on the flow velocity

\begin{tabular}{|l|l|l|l|}
\hline Volume Flow Rate $\left[\mathrm{m}^{3} / \mathrm{s}\right]$ & 30 & 40 & 50 \\
\hline Pressure losses $[\mathrm{Pa}]$ & 155 & 272 & 434 \\
\hline
\end{tabular}

The total pressure loss is understood as a sum of pressure losses through all the construction components which are defined by the use of the relation:

$$
\Delta p=\sum_{i} \frac{1}{2} \cdot \rho \cdot V_{i}^{2} \cdot \zeta_{i}
$$

Here $\rho$ is the gas density, taken to be $1.185 \mathrm{~kg} / \mathrm{m}^{3}, V_{i}$ is the gas velocity and $\zeta_{i}$ is the pressure loss coefficient within the construction components respectively.

Based on that, the total pressure loss coefficient $\zeta$ can be defined as

$$
\Delta p=\frac{1}{2} \cdot \rho \cdot V^{2} \cdot \zeta
$$

where $V$ is the operating velocity. Eventually, the THM wind tunnel is characterized with a total pressure loss coefficient of about 0.29 .

Once the total pressure loss has been fixed, the main power $P$ of the plant, needed to maintain the flow running inside the wind tunnel at a constant speed, may be assessed as

$$
\mathrm{P}=\Delta p \cdot \frac{\dot{\mathrm{Q}}}{\eta} \text {. }
$$

Here $\dot{Q}$ stays for the volumetric flow rate and $\eta$ is the fan efficiency. At a typical fan efficiency value of about $50 \%$, the fan power needed is specified to be of about $43.5 \mathrm{~kW}$. One has to mention that the figure given should be increase by $10 \%$ to account the pressure loss within the settling chamber and the dissipations as well as. 
The velocity distribution within the cross section of the nozzle exit is presented in Fig. 3 at the volume flow rate of $50 \mathrm{~m} / \mathrm{s}$. The velocity uniformity is estimated to be of about $3 \%$ within the core.

The flow structure depending on the swirl momentum imposed have been also studied. The rotational motor speed of $1000 \mathrm{rpm}$ has been assumed. So that three different scenarios have been simulated: $8 \mathrm{rev} / \mathrm{s}, 16 \mathrm{rev} / \mathrm{s}$ and $32 \mathrm{rev} / \mathrm{s}$ respectively. While the swirl impact affects only little the total pressure losses, it significantly modifies the flow structure within the test section.

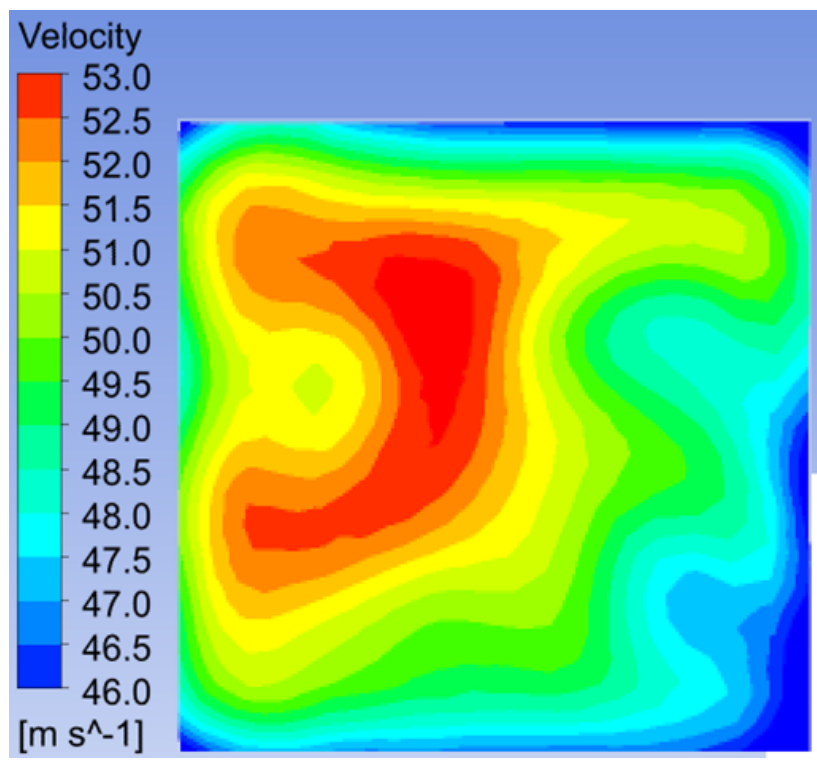

Fig. 3. Velocity distribution within the nozzle exit at volume flow rate of $50 \mathrm{~m}^{3} / \mathrm{s}$

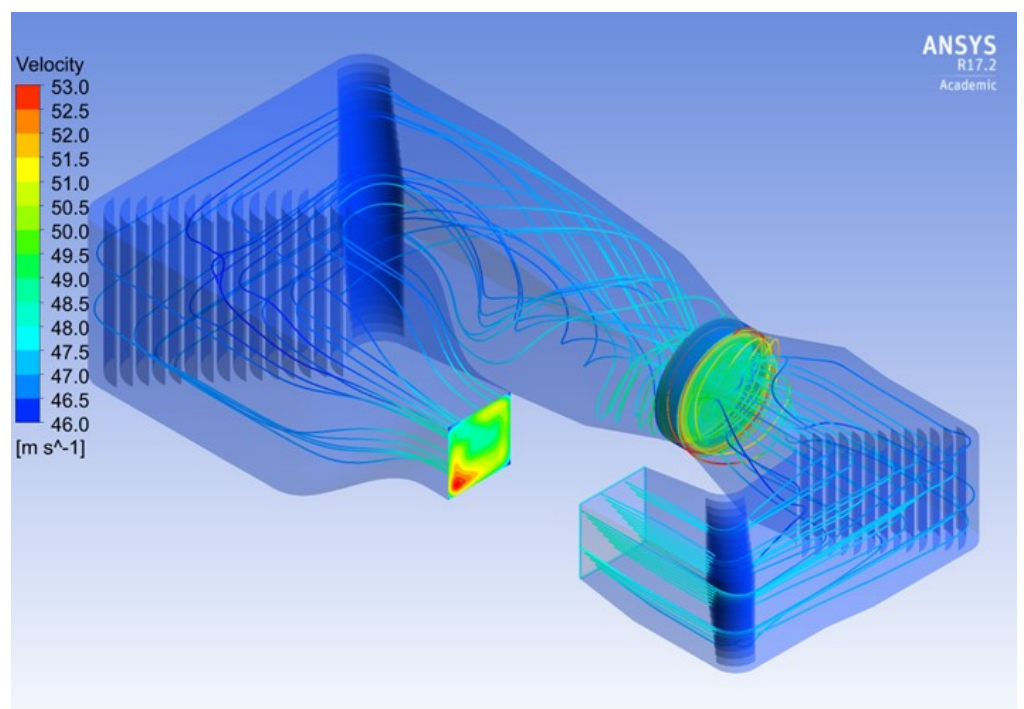

Fig. 4. Streamlines and velocity distribution within the wind tunnel at volume flow rate of $50 \mathrm{~m}^{3} / \mathrm{s}$ and $16 \mathrm{rev} / \mathrm{s}$ rotational impeller speed. 
To fully understand the flow structure, the velocity components in horizontal and vertical direction of the nozzle exit also have been studied. The streamlines and the velocity distribution within the wind tunnel at volume flow rate of $50 \mathrm{~m}^{3} / \mathrm{s}$ and $16 \mathrm{rev} / \mathrm{s}$ rotational impeller speed are given in Fig. 4, while the velocity components distribution within the cross section of the nozzle exit are shown in Fig. 5.

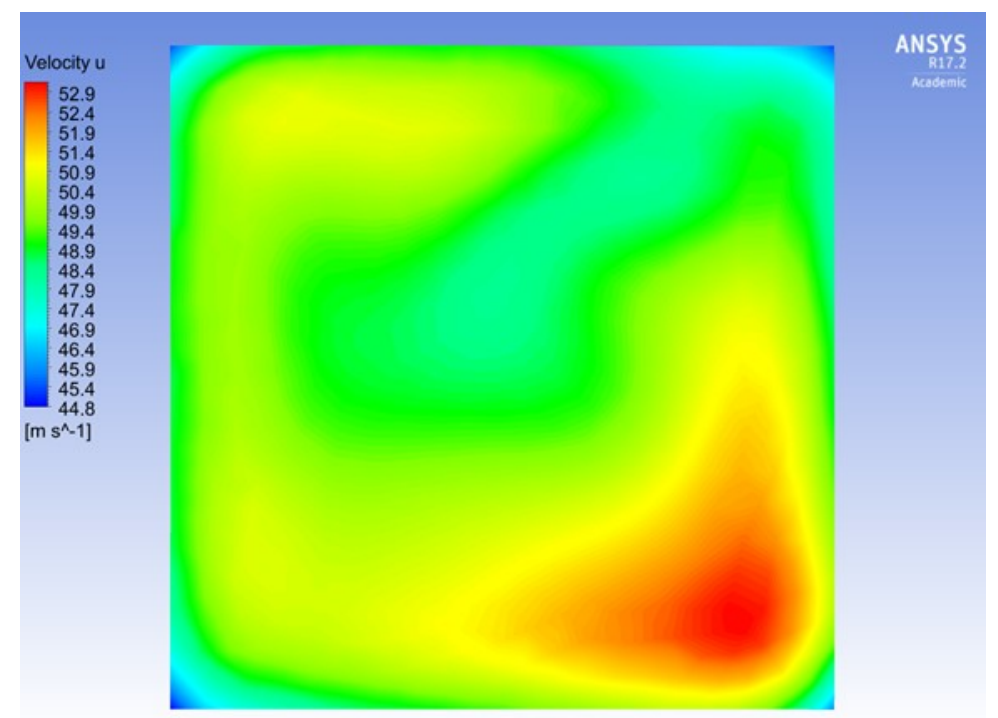

(a)

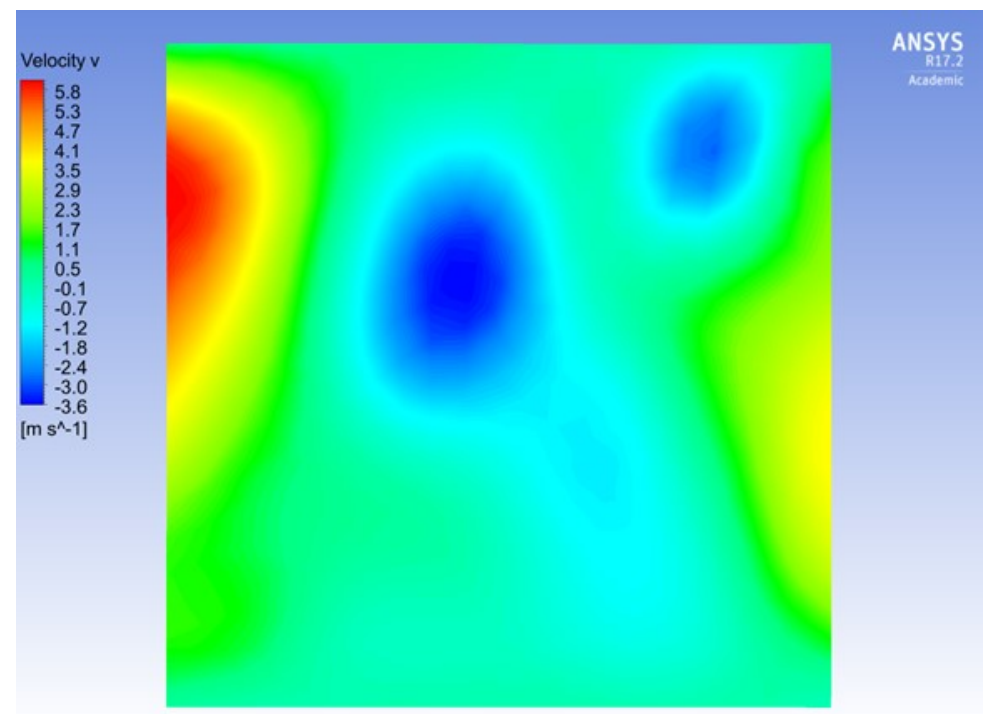

(b) 


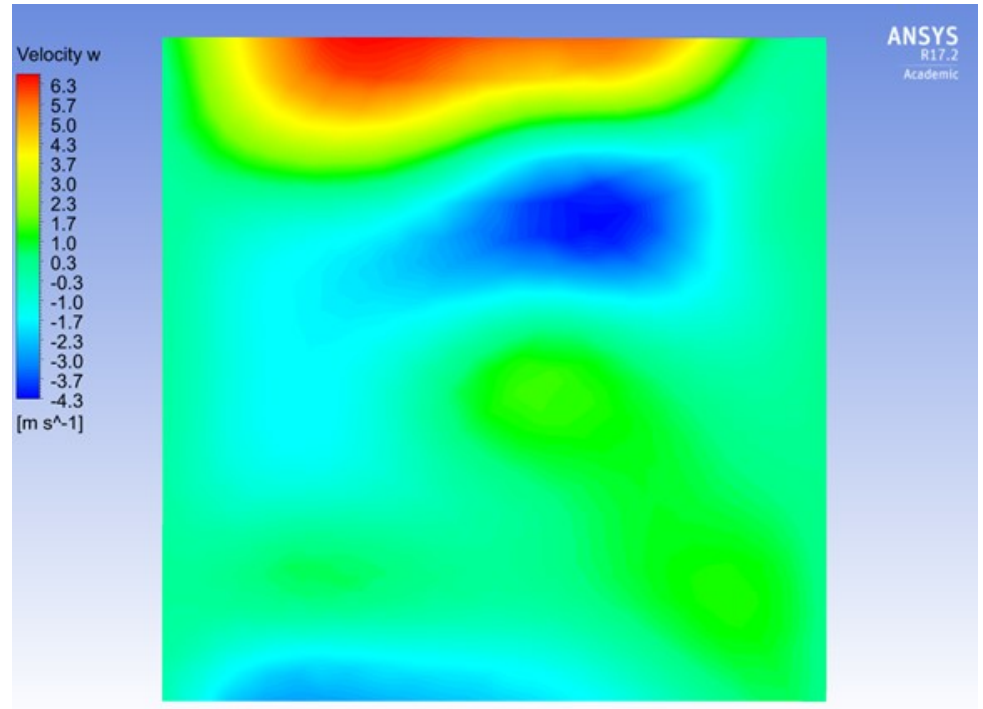

(c)

Fig. 5. Velocity components distribution within the cross section of the nozzle exit at volume flow rate of $50 \mathrm{~m}^{3} / \mathrm{s}$ and $16 \mathrm{rev} / \mathrm{s}$ rotational impeller speed: (a) velocity component in the direction perpendicular to the cross section exit; (b) velocity components in the horizontal direction; (c) velocity components in the vertical direction.

\section{Wind tunnel validation}

After wind tunnel has been built at the THM in Friedberg the specification has been validated by L. Feucht, D. Krön and S. Neumann [6]. The measurements have been made at three cross

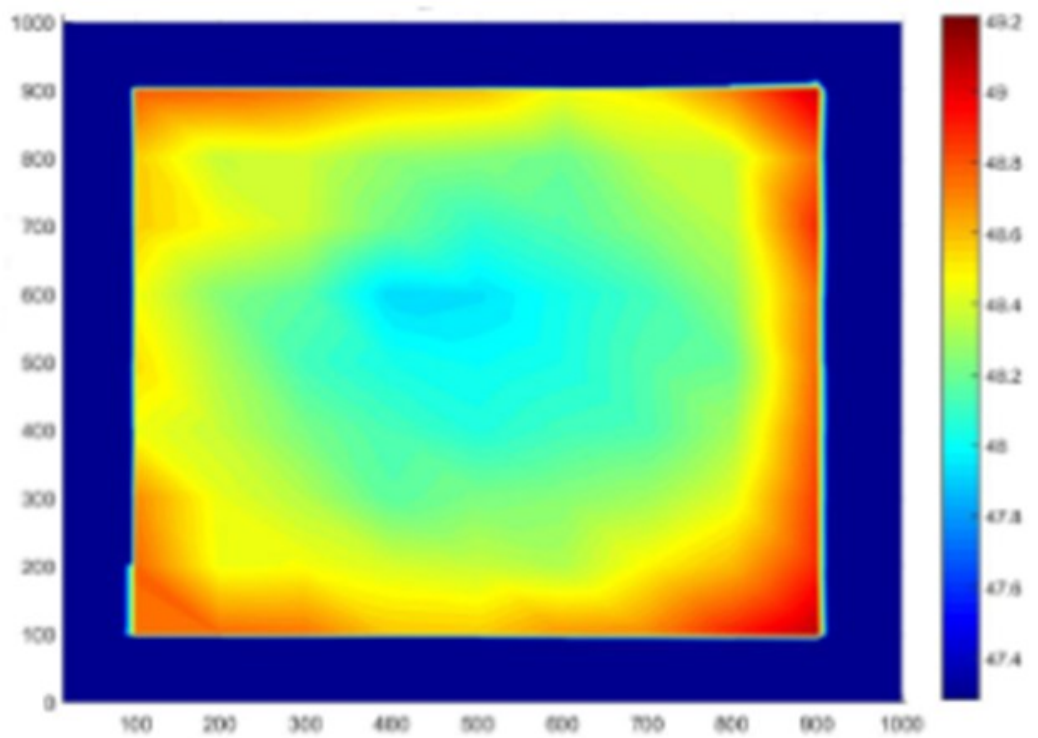

Fig. 6. Velocity distribution at the cross section with a distance of $0.4 \mathrm{~m}$ from the nozzle exit and a volume flow rate of $50 \mathrm{~m}^{3} / \mathrm{s}$ 
sections of the test section with distances to the nozzle exit of $0.4 \mathrm{~m}, 0.8 \mathrm{~m}$ and $1.2 \mathrm{~m}$ respectively. The specified maximal velocity of $50 \mathrm{~m} / \mathrm{s}$ was reached and the flow characteristics fulfilled the specification given (s. Fig. 6).

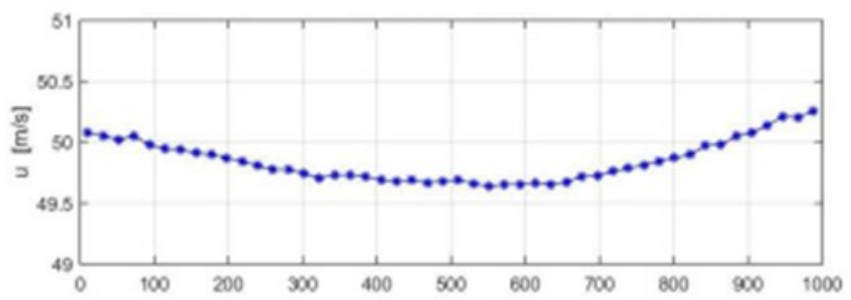

(a)

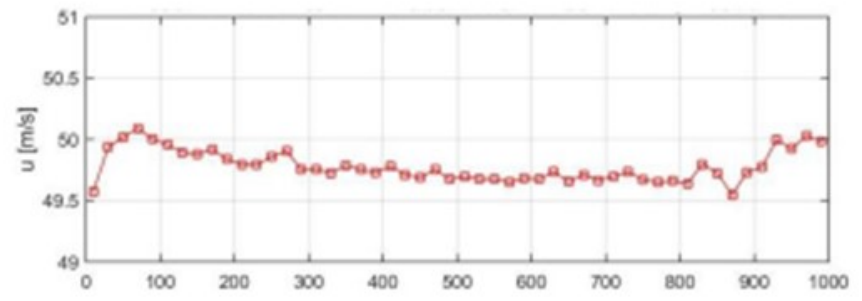

(b)

Fig. 7. Measured velocity at the middle of cross section of the test section at a distance of 0.4 from the nozzle exit as a function of the distance to the nozzle wall and volume flow rate of $50 \mathrm{~m}^{3} / \mathrm{s}$ : (a) in horizontal direction; (b) in vertical direction.

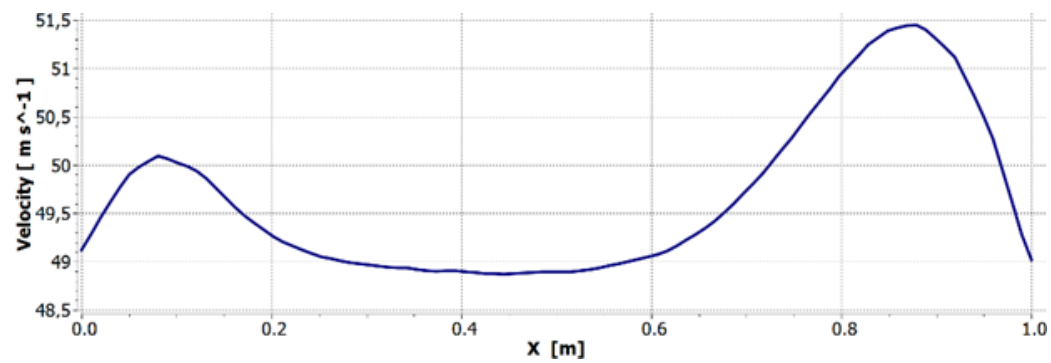

(a)

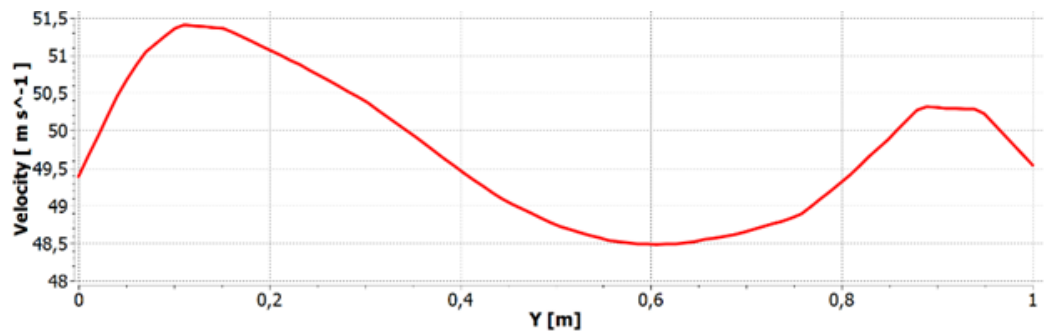

(b)

Fig. 8. Velocity at the middle of the nozzle cross section as a function of the distance to the nozzle wall and volume flow rate of $50 \mathrm{~m}^{3} / \mathrm{s}$ : (a) in horizontal direction; (b) in vertical direction. 
The data predicted have been compared with the experimental data and a good correlation has been found (s. Fig. 7 and Fig. 8 where the velocity at the middle of the nozzle cross section as a function of a distance to the nozzle wall is given). The discrepancies observed can be attributed to the specifics of the measurement. The velocity of the flow has been calculated at the exit of the tunnel taking into account the wall effects, however, the measurements have been carried out somewhere in the middle of the test section. The wall effects, affecting the flow in the tunnel, are there already negligible.

\section{References}

1. P. Branshaw, R. C. Pankhurst, Progress in aeronautical sciences, 6, 1 (1964)

2. W. H. Rae, A. POPE, A. Low-speed wind tunnel testing (2nd edn. John Wiley \& sons. 1984)

3. B. Lindgren, A.V. Johansson, Design and evaluation of a low-speed wind-tunnel with expanding corners (Technical Reports from Royal Institute of Technology, Departments of Mechanics, SE-100, 44 Stockholm, Sweden 2002)

4. M.A.G. Hernández, A.I.M. López., A.A. Jarzabek, J.M.P. Perales, Y., Wu, S. Xiaxiao, Design methodology for a quick and low-cost wind tunnel, Chapter 1 in Wind Tunnel Designs and Their Diverse Engineering Applications (Edited by N. A. Ahmed, ISBN 978-953-51-1047-7 (Open Acces) 2013)

5. ANSYS CFX-Solver Theory Guide, Inc. Release 14.5, Southpointe October (2012)

6. D. Krön, Messung und Bewertung der Strömungsturbulenz im Freistrahl des $1 \times 1 \mathrm{~m}^{2}$ Windkanals Göttinger Bauart GWK der THM (Projektarbeit 2015) 